\title{
Uniqueness for a wave propagation inverse problem in a half space
}

\author{
Matti Lassas ${ }^{1}$, Margaret Cheney ${ }^{2}$ and Gunther Uhlmann ${ }^{3}$
}

\begin{abstract}
This paper considers an inverse problem for wave propagation in a perturbed, dissipative half-space. The perturbation is assumed to be compactly supported. This paper shows that in dimension three, the perturbation is uniquely determined by knowledge of the Dirichlet-to-Neumann map on an open subset of the boundary.
\end{abstract}

\section{Introduction}

In this paper, we consider an inverse problem for wave propagation modeled by the equation

$$
\left(\nabla^{2}+q(x)\right) u(x)=0,
$$

where $x$ is in $\mathbf{R}^{3}$ and $q$ can be complex-valued. This equation arises in the propagation of electromagnetic [1], [3] and acoustic [5], [8] waves.

\footnotetext{
${ }^{1}$ Rolf Nevanlinna Institute, P. O. Box 4, FIN-00014 University of Helsinki, Finland. This research was supported by Finnish Academy project 37692 .

${ }^{2}$ Institute for Mathematics and Its Applications, University of Minnesota, Minneapolis, MN 55455; permanent address: Department of Mathematical Sciences, Rensselaer Polytechnic Institute, Troy, NY 12180. This research was supported in part by RPI, the IMA, ONR, and NSF.

${ }^{3}$ Department of Mathematics, University of Washington, Seattle, WA 98195. This research was partly supported by NSF and ONR.
} 
We consider the problem in the half-space $x_{3}<0$. In the lower half-space, $q(x)$ differs from the constant $q_{-}$only in a region of compact support. Here the imaginary part of $q(x)$ and $q_{-}$are assumed to be non-negative.

For this wave propagation problem we consider an inverse boundary value problem for the lower half-space. In particular, we specify Dirichlet data on the top surface:

$$
\left.u\right|_{x_{3}=0}=f,
$$

together with an outgoing radiation condition in the lower half-space. We assume that the boundary data $f$ is in the Sobolev space $H^{1 / 2}$ defined by

$$
H^{s}=\left\{f: \int|\hat{f}(\xi)|^{2}\left(1+|\xi|^{2}\right)^{s / 2} d \xi<\infty\right\} .
$$

For boundary data in this space, it is known that the boundary value problem (1), (2), together with radiation conditions, has a unique solution [1].

Thus the normal derivative $\partial u / \partial x_{3}$ on the surface $x_{3}=0$ is uniquely determined. The mapping from $H^{1 / 2}$ to $H^{-1 / 2}$

$$
\Lambda:\left.\left.u\right|_{x_{3}=0} \mapsto \frac{\partial u}{\partial x_{3}}\right|_{x_{3}=0}
$$

is called the Dirichlet-to-Neumann map. Such maps have been used a great deal recently in the study of inverse problems [13], [7], [10], [9], [11]. Knowledge of the Dirichlet-to-Neumann map is equivalent, in a certain sense, to scattering data [1].

The inverse boundary value problem is to determine $q(x)$ in the lower halfspace from knowledge of $\Lambda$. In the case in which the Dirichlet-to-Neumann map is defined on the boundary of a compact region, it is known [13] that knowledge of $\Lambda$ uniquely determines $q(x)$. The purpose of this paper is to extend this result to the half-space geometry. For the case in which $q$ is purely real, the half-space uniqueness question was studied in the unpublished manuscripts [14] and [4]. Here we consider the complex case.

\section{The theorem and proof}

We consider two equations of the form (1). We denote the two qs by $q_{1}$ and $q_{2}$, and we will use subscripts to denote the corresponding associated solutions, Green's functions, Dirichlet-to-Neumann maps, etc.. 
We assume that the general region of space containing the possible perturbations $q_{1}$ and $q_{2}$ is known. We denote by $B$ a large open set containing the supports of $q_{1}$ and $q_{2}$. We also assume that the perturbation is strictly contained in the lower half-space, so that the closure of $B$ does not intersect the boundary $x_{3}=0$.

The proof of the theorem will make use of the Dirichlet Green's function, which can be defined as follows by the method of images. We use a tilde to denote the image point, so that $\tilde{y}$ is the point obtained by reflecting $y$ across the $y_{3}=0$ plane. First we recall that the free-space outgoing Green's function corresponding to the medium parameters in the lower half-space is

$$
G^{0}(x, y)=\frac{e^{i \sqrt{q-}|x-y|}}{4 \pi|x-y|} .
$$

The full-space outgoing Green's function in the presence of a perturbation in the medium is then the unique solution [1] of the Lippmann-Schwinger equation $G=G^{0}+\int G^{0}\left(q-q_{-}\right) G$. For a source located at the image point $\tilde{y}$, the corresponding quantities are $\tilde{G}^{0}(x, y)=G^{0}(x, \tilde{y})$ and $\tilde{G}$, which satisfies $\tilde{G}=\tilde{G}^{0}+\int G^{0}\left(q-q_{-}\right) \tilde{G}$. Finally we define the Dirichlet Green's function $G^{D}$ to be the difference of the perturbed free-space Green's functions: $G^{D}=$ $G-\tilde{G}$. We note that $\tilde{G}^{D}$ satisfies the integral equation

$$
G^{D}(x, y)=G^{0}(x, y)-\tilde{G}^{0}(x, y)+\int G^{0}(x, z)\left(q(z)-q_{-}\right) G^{D}(z, y) d z
$$

This Dirichlet Green's function automatically satisfies the condition

$$
\left.G^{D}(x, y)\right|_{x_{3}=0}=0 .
$$

Moreover, from equation (5) it follows that Green's function $G^{D}(\cdot, y)$ satisfies the radiation condition

$$
\lim _{|x| \rightarrow \infty}|x|\left(\frac{\partial}{\partial|x|}-i k\right) G^{D}(x, y)=0 .
$$

Proposition 2.1 The kernel of the Dirichlet-to-Neumann map $\Lambda$ is

$$
\left.\frac{\partial}{\partial x_{3}} \frac{\partial}{\partial y_{3}} G^{D}(x, y)\right|_{x_{3}=0, y_{3}=0} \text {. }
$$


Proof. We apply the divergence theorem and radiation condition (7) to $\int\left(u \nabla^{2} G^{D}-G^{D} \nabla^{2} u\right)=u$, where $u$ denotes a solution of (1), (2), with radiation conditions in the lower half-space.

We denote by $R_{-}^{3}$ the lower half-space $\left\{x: x_{3}<0\right\}$, and by $A$ the set $R_{-}^{3} \backslash \bar{B}$. In the following we say that $\Lambda_{1}=\Lambda_{2}$ on some open subset $\Gamma$ of the boundary $x_{3}=0$ if the kernels of the operators coincide on $\Gamma \times \Gamma$, i.e.,

$$
\left.\frac{\partial}{\partial x_{3}} \frac{\partial}{\partial y_{3}} G_{1}^{D}(x, y)\right|_{x_{3}=0, y_{3}=0}=\left.\frac{\partial}{\partial x_{3}} \frac{\partial}{\partial y_{3}} G_{2}^{D}(x, y)\right|_{x_{3}=0, y_{3}=0}
$$

for $x$ and $y$ in $\Gamma$.

Theorem 2.1 Suppose the set $B$ containing the supports of $q_{1}-q_{-}$and $q_{2}-q_{-}$is strictly contained in the lower half-space. If $\Lambda_{1}=\Lambda_{2}$ on some open subset $\Gamma$ of the boundary $x_{3}=0$, then $q_{1}=q_{2}$.

Proof. The proof involves a series of five lemmas. The first two show that knowledge of the Dirichlet-to-Neumann map suffices to determine the Dirichlet Green's function outside the perturbation in $q$. The third and fourth lemmas establish a version of the Green's theorem identity that is often used for uniqueness arguments. The last lemma, which is roughly based on the uniqueness proof in [6], shows that linear combinations of the Dirichlet Green's functions can be used to approximate the Sylvester-Uhlmann solutions.

Lemma 2.2 Suppose the hypotheses of the theorem are satisfied. Then $\partial_{y_{3}} G_{1}^{D}(x, y)=\partial_{y_{3}} G_{2}^{D}(x, y)$ for $y$ in $\Gamma$ and $x$ in $A$.

Proof. For a fixed $y$ in $\Gamma$, we denote by $F_{i}(x)$ the function $\partial_{y_{3}} G_{i}^{D}(x, y)$ for $i=1,2$. Each $F_{i}$ satisfies the Helmholtz equation $\left(\nabla^{2}+q_{-}\right) F_{i}(x)=0$ in the set $A$ away from the perturbation. Because $\Lambda_{1}=\Lambda_{2}$ on $\Gamma$, from (9) we have that the normal derivatives of $F_{i}$ coincide there: $\partial_{x_{3}} F_{1}(x)=\partial_{x_{3}} F_{2}(x)$ for $x$ on $\Gamma$. Moreover, by taking $y_{3}$ derivatives of both sides of the boundary condition (6) we see that for $x$ on $\Gamma, F_{1}(x)=F_{2}(x)=0$. Thus $F_{1}$ and $F_{2}$ satisfy the same unperturbed Helmholtz equation outside $\bar{B}$, and have identical Cauchy data on $\Gamma$. The Holmgren uniqueness theorem can be used to show that $F_{1}$ and $F_{2}$ are identical in a neighborhood of $\Gamma$. From this the claim follows from the unique continuation principle [2]. 
Lemma 2.3 Suppose the hypotheses of the theorem are satisfied. Then $G_{1}^{D}(x, y)=G_{2}^{D}(x, y)$ for $x$ in $A$ and $y$ in $A \backslash\{x\}$.

Proof. Let $x$ in $A$ be fixed. Then $G_{i}^{D}(x, y)$ satisfies the Helmholtz equation $\left(\nabla^{2}+q_{-}\right) G_{i}^{D}(x, \cdot)=0$ in the set $A \backslash\{x\}$. By the boundary condition (6) and reciprocity, $G_{i}^{D}(x, y)=0$ for $y$ in $\Gamma$. By Lemma $2.2, \partial_{y_{3}} G_{1}^{D}(x, y)=$ $\partial_{y_{3}} G_{2}^{D}(x, y)$ for $y$ in $\Gamma$. Thus $G_{1}^{D}$ and $G_{2}^{D}$ satisfy the homogeneous Helmholtz equation in $A \backslash\{x\}$ and have the same Cauchy data on $\Gamma$. Again, by the Holmgren uniqueness theorem $G_{1}^{D}(x, \cdot)$ and $G_{2}^{D}(x, \cdot)$ are identical in a neighborhood of $\Gamma$. Thus by the unique continuation principle they coincide everywhere outside $\bar{B} \cup\{x\}$.

Lemma 2.4 For $x$ in $B$, and $y$ and $z$ in $A$,

$$
\begin{aligned}
& \int_{B}\left(q_{i}-q_{j}\right)(x) G_{i}^{D}(x, y) G_{j}^{D}(x, z) d x \\
& =\int_{\partial B}\left(G_{i}^{D}(x, y) \partial_{\nu_{x}} G_{j}^{D}(x, z)-G_{j}^{D}(x, z) \partial_{\nu_{x}} G_{i}^{D}(x, y)\right) d S_{x},
\end{aligned}
$$

where $\partial_{\nu_{x}}$ denotes differentiation with respect to the outward unit normal to $B$.

Proof. This follows from the divergence theorem.

Lemma 2.5 Suppose the hypotheses of the theorem are satisfied. Then for $x$ in $B$, and $y$ and $z$ in $A$,

$$
\int_{B}\left(q_{1}-q_{2}\right)(x) G_{1}^{D}(x, y) G_{2}^{D}(x, z) d x=0 .
$$

Proof. From Lemma 2.4 with $i=1, j=2$ we have

$$
\begin{aligned}
& \int_{B}\left(q_{1}-q_{2}\right)(x) G_{1}^{D}(x, y) G_{2}^{D}(x, z) d x \\
& =\int_{\partial B}\left(G_{1}^{D}(x, y) \partial_{\nu_{x}} G_{2}^{D}(x, z)-G_{2}^{D}(x, z) \partial_{\nu_{x}} G_{1}^{D}(x, y)\right) d S_{x} .
\end{aligned}
$$

However, by Lemma $2.3, G_{1}^{D}=G_{2}^{D}$. By applying Lemma 2.4 in the case $i=j$ see that the right hand side of (11) vanishes and thus we obtain (10). 
Lemma 2.6 The span of the set $U=\left\{\left.G^{D}(\cdot, y)\right|_{B}: y\right.$ in an open subset of A\} is dense in the set $V=\left\{\left.u\right|_{B}:\left(\nabla^{2}+q\right) u=0\right\}$.

Proof. Suppose there exists an $f$, supported in $B$, that is orthogonal to all $G^{D}$ in $U$, i.e., $\int_{B} G^{D}(x, y) f(x) d x=0$ for all $y$ in an open subset of $A$. Let $w(y)=\int_{B} G^{D}(x, y) f(x) d x$. Then $\left(\nabla^{2}+q\right) w=f$. By assumption, $w$ is identically zero in an open subset of $A$, and by unique continuation [2], must be zero everywhere in $A$.

This enables us to show as follows that $f$ must also be orthogonal to every solution $u$ in $V$ :

$$
\int_{B} u f=\int_{B} u\left(\nabla^{2}+q\right) w=\int_{B} w\left(\nabla^{2}+q\right) u=0 .
$$

Here the second equality follows because $w$ vanishes outside $B$, which implies the vanishing of the boundary terms arising from integrating by parts.

The proof of the theorem concludes with the observation that Lemma 2.6 guarantees that we can use linear combinations of the functions $G^{D}$ to approximate the Sylvester-Uhlmann solutions [13], which are of the form

$$
\psi(x, \zeta)=e^{i \zeta \cdot x}+O(1 /|\zeta|)
$$

for complex vectors $\zeta$ such that $\zeta \cdot \zeta=q_{-}$. Then for any real $\xi$, we use $G_{j}^{D} \mathrm{~s}$ to approximate $\psi_{j}=\psi\left(x, \zeta_{j}\right)$ with $\zeta_{1}+\zeta_{2}=\xi$, and thus on the left side of (10) obtain the Fourier transform of $q_{1}-q_{2}$. Since this Fourier transform can be made arbitrarily close to zero, $q_{1}=q_{2}$.

\section{References}

[1] M. Cheney and D. Isaacson, "Inverse Problems for a Perturbed Dissipative Half-Space", Inverse Problems 11, 856-888 (1995).

[2] D. Colton and R. Kress, Inverse Acoustic and Electromagnetic Scattering Theory, Springer, New York, 1992.

[3] J.D. Jackson, Classical Electrodynamics, 2nd Ed., Wiley, New York, 1975 . 
[4] G. Eskin and J. Ralston, "Inverse coefficient problems in perturbed halfspaces", preprint, 1997.

[5] A. Kirsch, “An Introduction To The Mathematical Theory Of Inverse Problems", Springer, New York, 1996.

[6] R. Melrose, "Geometric Scattering Theory", Cambridge University Press 1995.

[7] A. Nachman, "Reconstructions from boundary measurements," Annals of Math. $\mathbf{1 2 8}$ (1988), pp. 531-556.

[8] F. Natterer and F. Wübbeling, "A propagation-backpropagation method for ultrasound tomography", Inverse Problems 11, 1225-1232 (1995).

[9] P. Ola, L. Päivärinta, and E. Somersalo, "An inverse boundary value problem in electrodynamics", Duke Math. J. 70 (1993) 617-653.

[10] E. Somersalo, M. Cheney, D. Isaacson, and E. Isaacson, "Layer Stripping: A Direct Numerical Method for Impedance Imaging," Inverse Problems, 7 (1991) 899-926 ; 8 (1992), 493.

[11] E. Somersalo, "Layer stripping for time-harmonic Maxwell's equations with fixed frequency", Inverse Problems 10 (1994) 449-466.

[12] J. Sylvester, “A convergent layer stripping algorithm for the radially symmetric impedance tomography problem", to appear, Comm. Partial Diff. Eqs.

[13] J. Sylvester and G. Uhlmann, "A uniqueness theorem for an inverse boundary value problem in electrical prospection", Comm. Pure Appl. Math. 39 (1986) 91-112; "A global uniqueness theorem for an inverse boundary value problem in electrical prospection", Ann. of Math., 125 (1987) 153-169; "Inverse boundary value problems at the boundarycontinuous dependence", Comm. Pure Appl. Math. 41 (1988) 197-221.

[14] R. Weder, "Uniqueness theorem for an inverse problem in geophysics", preprint, Universidad Nacional Autónoma de México. 